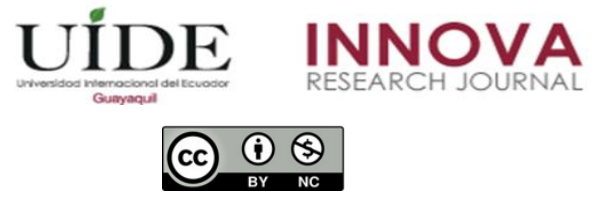

INNOVA Research Journal, ISSN 2477-9024

(Enero-Abril 2020). Vol. 5, No.1 pp. 129-137

DOI: https://doi.org/10.33890/innova.v5.n1.2020.1020

URL: http://revistas.uide.edu.ec/index.php/innova/index

Correo: innova@uide.edu.ec

\title{
IGV justo y la liquidez en una empresa de servicios, Lima 2017
}

\section{Fair IGV and liquidity in a services company, Lima 2017}

Nilda Rojas Campos

https://orcid.org/0000-0002-7837-9319

Universidad Norbert Wiener, Perú

Autor por correspondencia: nildarc04@gmail.com

Fecha de recepción: 15 de junio del 2019 - Fecha de aceptación: 07 de noviembre del 2019

\section{Resumen}

La investigación tiene la finalidad de analizar la incidencia del IGV justo en la liquidez de una empresa de servicios ubicada en Lima - Perú, periodo 2017, periodo en el cual se dio vigencia a la Ley 30524-IGV Justo. El estudio se desarrolló bajo un enfoque cualitativo y con la aplicación del análisis documental al estado de situación financiera de la empresa, y la entrevista a los informantes, por consiguiente, se recurrió al apoyo del software Atlas.ti para la triangulación de data. En ese sentido se puede indicar que la prolongación del pago del Impuesto General a las Ventas hasta 90 días, tiene una repercusión en la liquidez de la empresa, acortando la brecha que existe entre la política de cobranza que brinda la empresa a sus clientes y el periodo que la entidad recaudadora emite para el pago del IGV. Asimismo, se detectó que la aplicación del IGV justo no fue $100 \%$ adecuada, debido a la falta de capacitación del personal contable frente a nuevas normativas.

Palabras claves: IGV justo; capital de trabajo; liquidez absoluta; liquidez corriente; impuesto

\begin{abstract}
The research has the purpose of analyzing the incidence of fair IGV in a services company liquidity, located in Lima- Peru, 2017 period, in which law $\mathrm{N}^{\circ}$ 30524- fair IGV was enforced. The study was developed under a qualitative approach, with the application of documentary analysis on the financial situation of the company, and interviews to informants, therefore, Atlas.ti software was required for data triangulation. To that effect, it can be stated that the 90 days-extension in the deadline for general sales tax payment has an impact on the company liquidity, shortening the existing gap between the collection policy provided by the company to its customers, and the IGV payment period that the public collecting entity establishes. Likewise, it was detected that the application of fair IGV was not $100 \%$ appropriate, due to the lack of training of the accounting staff, about new regulations.
\end{abstract}

Key words: fair IGV; working capital; absolute liquidity; current liquidity; tax 


\section{Introducción}

El IGV o también conocido en otros países como IVA, es uno de los impuestos con mayor repercusión en cuanto a recaudación (Chirinos, 2009). Por ello, se ha convertido en una tarea primordial para el estado combatir la evasión tributaria y brindar las mayores facilidades a los empresarios para que cumplan con su deber tributario (Carvalho, 2013).

En algunos países de Latinoamérica como Chile y Argentina, se han implementado leyes a favor de las Mypes, que han permitido prolongar el pago del IVA. En Perú, un país con miras a ser miembro de la OCDE, en los últimos años ha reforzado su sistema tributario originando y mutando normas; claro ejemplo es la Ley del IGV justo con vigencia en el 2017. Dicha ley tiene el objetivo que las mypes, empresas que están tratando de ganarse un espacio en el mercado comercial, puedan cumplir con el pago de sus tributos, que por razones de realizar ventas al crédito no cumplen o recurren muchas veces a su capital de trabajo o entidades financieras (Mostacero, 2018).

El análisis de la repercusión del IGV justo en la liquidez, se llevó a cabo en una empresa de servicios, que cuenta con una política de cobranza de 30 hasta 90 días, en la cual los clientes no respetan dicha política. Pese a ello, tiene la obligación de cumplir con el pago del impuesto por un ingreso que aún no ha percibido, además la entidad escasea de un personal encargado de créditos y cobranzas.

Un personal capacitado es primordial en una entidad para que las normativas tributarias tengan una correcta aplicación o estar alertas a posibles infracciones. Asimismo, aplicar una auditoría a la gestión es importante para detectar anomalías y estar preparados ante fiscalizaciones futuras.

\section{IGV justo}

Beneficio que tiene permitido a las micro y pequeñas empresas prolongar las fechas de pago del IGV hasta 90 días, siendo indiferente a la fecha de declaración mensual del impuesto publicado por la entidad recaudadora. Uno de los requisitos primordiales para poder acogerse, es que sus ingresos anuales no excedan las 1700 Unidades Impositivas tributarias (SUNAT, 2017).

\section{Liquidez}

Capacidad de afrontar o finiquitar con el activo corriente aquellas deudas pactadas dentro de los 12 meses (Puente, 2006; Flores,2016). Para ello, existen índices de medición de liquidez que permiten a la empresa realizar toma de decisiones frente al resultado obtenido, eliminando aquellas dolencias que no permite obtener buena salud en la liquidez o mejorar los puntos débiles (Albisetti, 2018; Brun, Larraga \& Moya, 2016).

\section{Objetivos}

\section{Objetivo General}


Analizar la incidencia del IGV justo en la liquidez en una empresa de servicios, Lima 2017

\section{Objetivos Específicos}

Analizar la incidencia del IGV justo en el capital de trabajo en una empresa de servicios, Lima 2017.

Analizar la incidencia del IGV justo en la liquidez corriente de una empresa de servicios, Lima 2017.

Analizar la incidencia del IGV justo en la liquidez absoluta de una empresa de servicios, Lima 2017.

\section{Método}

Se aplicó un enfoque cualitativo, desarrollando el caso particular IGV justo en el ámbito de la liquidez. De acuerdo a la investigación, se determinó que a partir de ello muchas empresas sufren las mismas dolencias, por lo cual, se aplicó el método inductivo (Bisquerra, 2009; Palella \& Martins, 2012). Asimismo, se desarrolló la entrevista a 3 informantes de la empresa y el análisis documental al estado de situación financiera (Ibáñez, 2015; Hernández, Fernández y Baptista,2014). Finalmente, con el apoyo del Atlas ti se realizó la triangulación como método de análisis.

\section{Resultados}

Se realizó entrevistas a 3 personales de la empresa, entrevistado 1 (Gerente general), entrevistado 2 (Contador) y entrevistado 3 (Asistente contable). Asimismo, se aplicó análisis documental al estado de situación financiera y reportes de récord de pago del Impuesto general a las ventas, del cual se obtuvieron los siguientes resultados: 


\begin{tabular}{|c|c|c|c|}
\hline \multicolumn{4}{|c|}{$\begin{array}{c}\text { ESTADO DE SITUACIÓN FINANCIERA } \\
\text { Al } 31 \text { de diciembre del } 2017 \\
\text { ( Expresado en Soles ) }\end{array}$} \\
\hline \multicolumn{2}{|l|}{ ACTIVO } & \multicolumn{2}{|l|}{ PASIVO Y PATRIMONIO } \\
\hline \multicolumn{2}{|l|}{ ACTIVO CORRIENTE } & \multicolumn{2}{|l|}{ PASIVO CORRIENTE } \\
\hline Efectivo y equivalente de efectivo & 35,998 & Sobregiros bancarios & \\
\hline Cuentas por cobrar comerciales-terc & 344,306 & Tributos por pagar & 32,870 \\
\hline \multicolumn{2}{|l|}{ Ctas./Cobrar accion./socios/pers/ger } & Cuentas por pagar comerciales- te reeros & 54,592 \\
\hline Cuentas por cobrar diversas-terc & 1,763 & Obligaciones Financieras corriente & 387,638 \\
\hline \multicolumn{2}{|l|}{ Serv. y otros contrata dos por anticip } & Cuentas por pagar diversas & 1,878 \\
\hline Materias aux.suministros, re puestos & 24,372 & TOTAL PASIVO CORRIENTE & 476,978 \\
\hline Activo Dife rido Corriente & 79,303 & & \\
\hline \multicolumn{2}{|l|}{ Creditos tributa rios Renta } & \multicolumn{2}{|l|}{ PASIVO NO CORRIENTE } \\
\hline TOTAL ACTIVO CORRIENTE & 485,741 & \multicolumn{2}{|l|}{ Cuentas por pagar comerciales- te reeros } \\
\hline \multicolumn{2}{|l|}{ ACTIVO NO CORRIENTE } & \multicolumn{2}{|l|}{$\begin{array}{l}\text { Cuentas p pagar accion, directores.soc. } \\
\text { Obligaciones Financieras no corriente }\end{array}$} \\
\hline Activo Dife rido & 85,244 & TOTAL PASIVO NO CORRIENTE & $1,049,401$ \\
\hline Inmuebles, ma quinaria y equipos(Neto) & $1,374,934$ & & \\
\hline \multirow[t]{4}{*}{ Activos Intangibles } & 1,718 & \multicolumn{2}{|l|}{ PATRIMONIO } \\
\hline & & Capital & 33,000 \\
\hline & & Resultados acumulados & 336,683 \\
\hline & & Utilidad (Pérdida) del ejercicio & 51,575 \\
\hline TOTAL ACTIVO NO CORRIENTE & $1,461,896$ & TOTAL PATRIMONIO & 421,258 \\
\hline TOTAL ACTIVO & $1,947,637$ & TOTAL PASIVO Y PATRIMONIO & $1,947,637$ \\
\hline
\end{tabular}

Figura 1. Estado de Situación Financiera

Al contar con una política de cobranza de 30 a 90 días y escasez de personal encargado de créditos y cobranzas, se detectó en el estado de situación financiera el alto importe en las cuentas por cobrar. Por esta razón, refleja que la entidad tiene que afrontar un impuesto general a las ventas por ingresos facturados pero aún no percibidos dentro de efectivo en caja. Debido a ello la entidad se acogió al IGV justo en febrero del 2017.

De acuerdo al análisis documental y al récord de pago del IGV, la entidad ha tenido que afrontar la determinación de la deuda tributaria basada en la facturación total de ventas, ascendentes a S/ 1'291,058.00; que de acuerdo a la tasa de IGV de 18\% se obtuvo un débito fiscal de S/ 232,390.00. En efecto, se aplicó un crédito fiscal S/ 152,931.00 obtenido de las compras realizadas durante el periodo. Cabe resaltar que mayo fue el mes con alta facturación, debido que su cliente potencial, una reconocida empresa en el rubro de producción y comercialización de cementos, obtuvo mayor demanda de proyectos.

Con respecto al nivel de incidencia del IGV en el capital de trabajo, al aplicar el ratio activo corriente menos pasivo corriente se obtuvo un resultado de $\mathrm{S} / 8,763.00$, es decir posterior a finiquitar las deudas que tenía dentro de los 12 meses se obtuvo dicho importe. Respecto a ello, en las entrevistas realizadas el entrevistado 3 indicó, el capital de trabajo permitió invertir, así como pagar algunas deudas que estaban programadas a largo plazo, sin embargo, otro de los entrevistados detalló, que mencionar aumento de capital gracias a la liquidez fue algo exagerado, debido que la ley no tuvo un $100 \%$ de provecho pues la vigencia de la ley era reciente. 


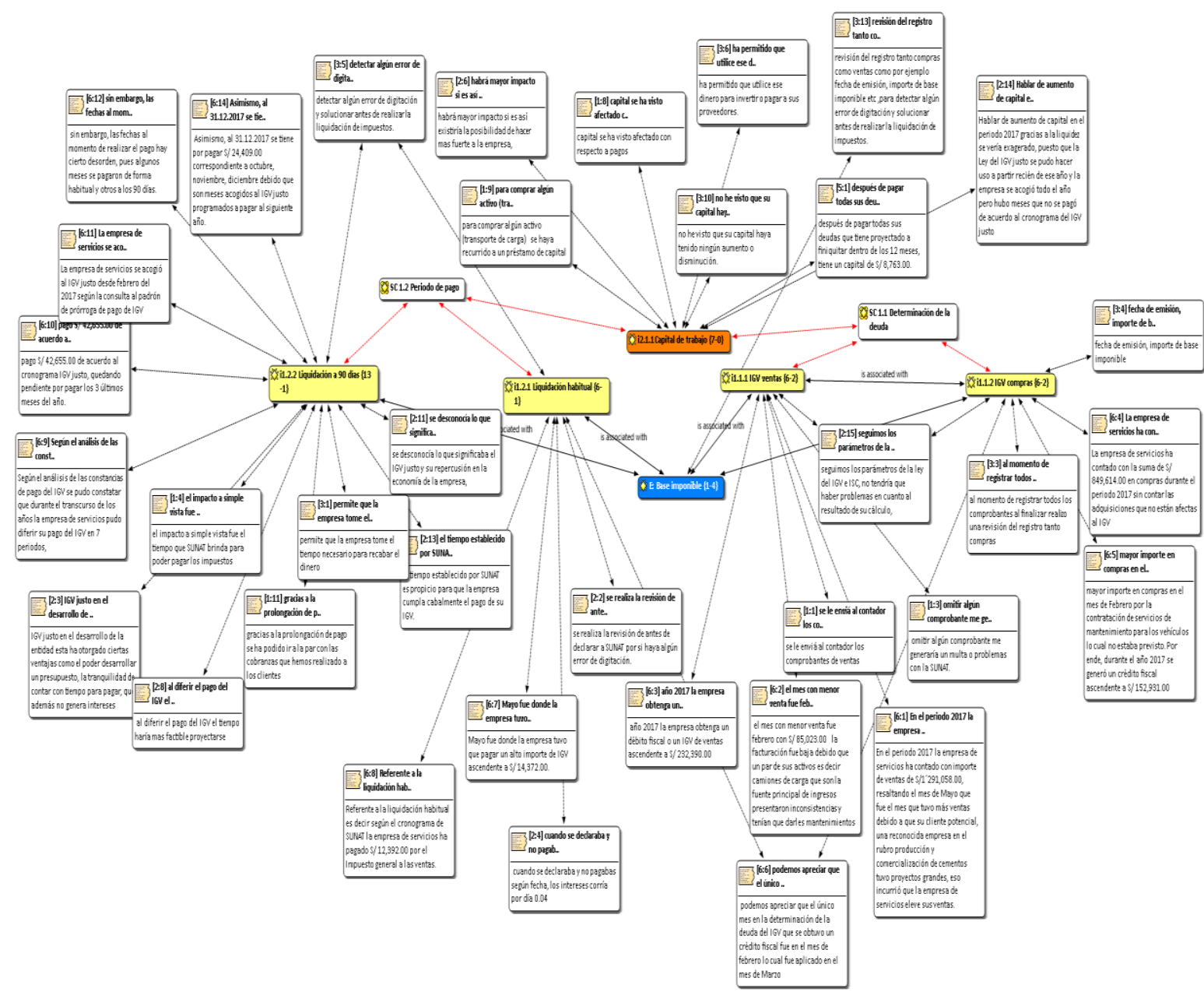

Figura 2. Triangulación del IGV justo y el capital de trabajo

En base a lo expuesto relacionado al Capital de trabajo, se recomienda analizar el destino del capital de trabajo y evitar los préstamos de capital. Asimismo, realizar Estados Financieros trimestral aplicando los índices de medición financiera que permita a gerencia tener un panorama de la situación para visualizar el impacto de la prórroga del IGV, además recomendar a gerencia que se relacione con el área contable.

En cuanto a la liquidez corriente se puede indicar que por cada 1 sol de deuda que tiene la empresa en su pasivo corriente, podrá afrontar con 1.02 soles, lo cual demuestra que la entidad tiene una situación estable y neutral, y puede cumplir con todas sus deudas a corto plazo solo haciendo uso de su activo corriente.

Por lo mencionado anteriormente, se verificó que la empresa al haber declarado sus impuestos mediante el PDT 621 acogiéndose al IGV justo, no ha respetado el cronograma de pago lo cual fue una de los factores para no obtener un mejor resultado en cuanto a la liquidez corriente.

Según el análisis de las entrevistas, las cifras plasmadas en el pasivo corriente en cuanto a impuestos a pagar, que derivan de los comprobantes declarados ante la entidad recaudadora, 
pasan por una revisión para verificar si siguen los parámetros de la Ley de Comprobantes de pago y la Ley del IGV e Impuesto selectivo al consumo; de esa manera permite a la empresa evitar que en futuras fiscalizaciones se le detecte errores y le apliquen multas. El encargado de ello es el asistente contable que también realiza las declaraciones mensuales.

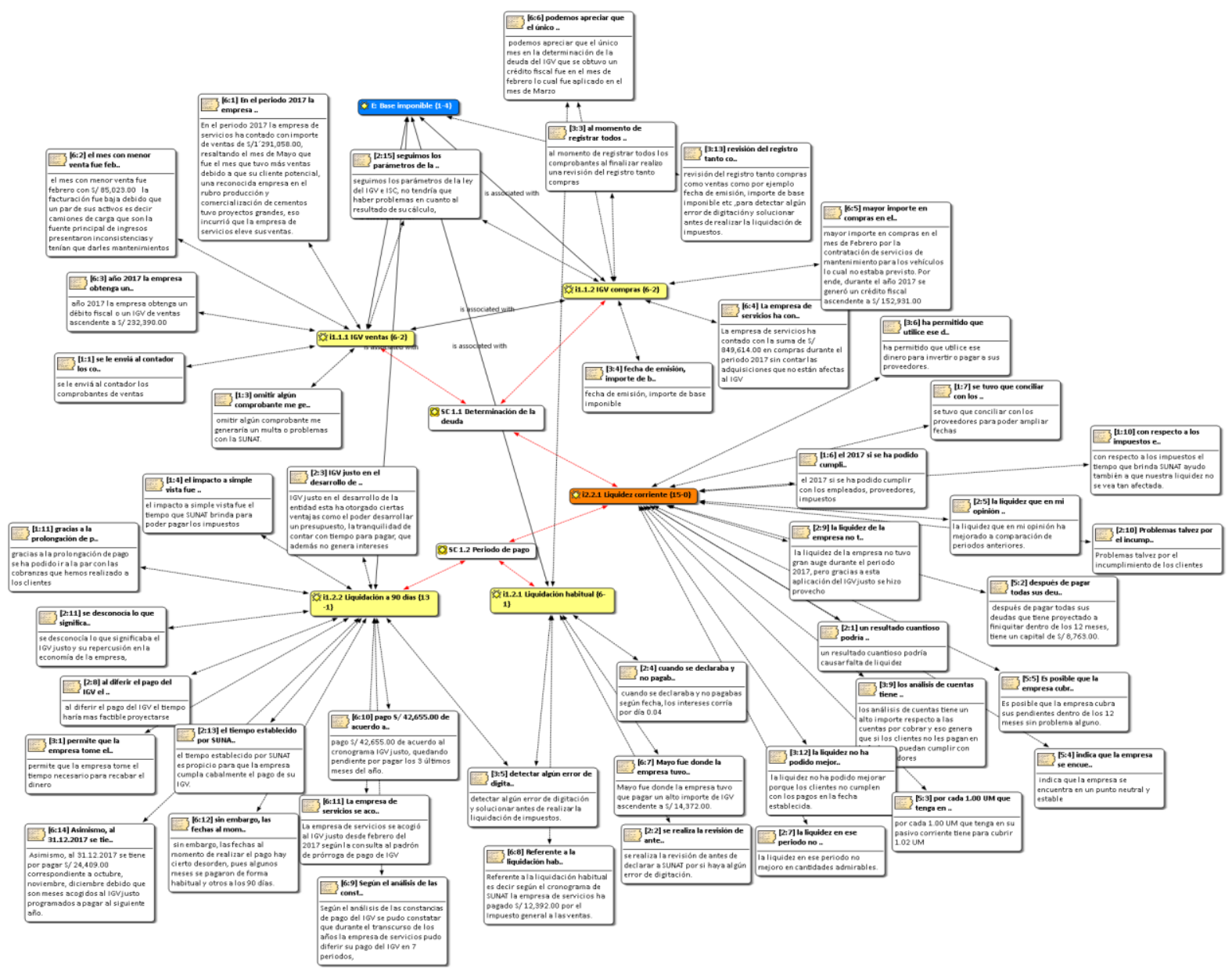

Figura 3. Triangulación del IGV justo y liquidez corriente

Por ser una de las empresas dentro del rango de las MYPES, tiende aún más a flaquear y no subsistir en el mercado debido a la falta de caja disponible en el preciso momento que lo requiere, esto se puede constatar en el resultado obtenido al aplicar el ratio de liquidez absoluta en el análisis documental. El ratio arrojó para el periodo 2017, 0.08, lo cual indica que la empresa lamentablemente tiene un riesgo frente a sus obligaciones a corto plazo si solo desea hacer uso de efectivo y equivalente de efectivo.

Por ello, la entidad tuvo que conciliar con sus proveedores para ampliar fechas de pago y así cumplir con sus obligaciones primordiales, como planilla e impuestos. El impuesto general a las ventas afectó a la empresa durante el periodo, debido que los clientes no pagaban en su fecha, además, en los primeros meses la empresa tuvo que afrontar de caja un IGV por ingresos que aún no percibían en efectivo. 
Octubre fue el mes más complicado, porque se detectó un desembolso para el pago de IGV de 2 periodos, consecuencia del desorden generado por el área de contabilidad, falta de asesoría y desinterés de gerencia. Caja afrontó un IGV ascendente a S/ 19,380.00 correspondiente al IGV mayo que fue prorrogado a octubre gracias al IGV justo, e IGV setiembre declarado de manera habitual.

Al respecto, es importante contar con un personal que se dedique a las cobranzas y seguimiento de la morosidad de los clientes, mediante correo y llamadas para respetar la política de cobranza, y así reducir las cuentas morosas y aumentar el efectivo disponible. Asimismo, establecer rangos de crédito a los clientes para evitar que la morosidad refleje índices de aumento.

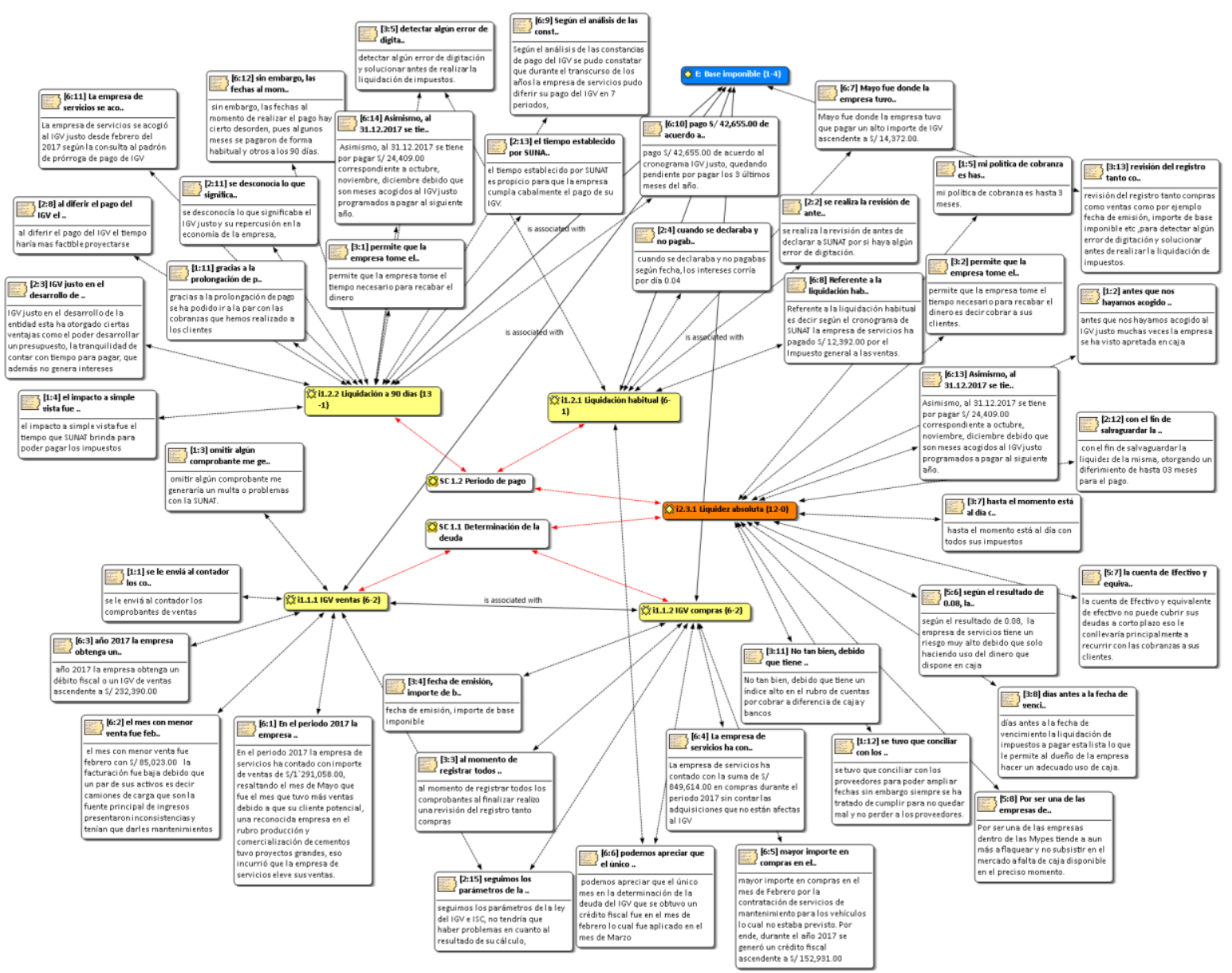

Figura 4 Triangulación del IGV justo y liquidez absoluta

\section{Conclusiones}

Existe un grado positivo de incidencia entre las 2 variables en estudio, sin embargo, la entidad no brinda capacitación sobre actualizaciones tributarias al personal contable, por ello el beneficio de prolongar el pago del IGV se aplicó de manera desordenada. Asimismo, se demuestra la falta de interés de Gerencia hacia las gestiones contable-tributaria. 
La empresa de servicios al término del 2017, a pesar que las unidades informantes no lo esperaban, arroja un capital de trabajo positivo al aplicar el ratio. La entidad está en la facultad de poder hacer uso de ese dinero para invertir o pagar alguna deuda de su pasivo no corriente.

En cuanto a la liquidez corriente, existe la capacidad de hacer frente a sus obligaciones a corto plazo, asimismo por acogerse al IGV justo tiene por afrontar el Impuesto General a las Ventas de los tres últimos meses del 2017, que asciende a S/ 24,409.00, en lo cual está en la capacidad haciendo uso de su activo corriente.

La empresa refleja la falta de efectivo inmediato para cubrir sus deudas a corto plazo, dentro de ellas el pago del Impuesto de IGV. La causa principal es la morosidad de sus clientes, por una indebida gestión de política de cobranza, por ello hay un alto importe en las Cuentas por cobrar. Las múltiples funciones que tienen el personal contable y gerencia que no corresponde a su puesto o cargo laboral, incurre que no haya una gestión adecuada de cobranza.

\section{Bibliografía}

Albisetti, R. (2018). Finanza empresarial: Estrategia, mercados y negocios estructurados. Bogotá: Pontificia Universidad Javeriana.

Bisquerra, R. (2009). Metodología de la investigación educativa. Madrid: La Muralla.

Bodie, Z., \& Merton, R. (2003). Finanzas. México: Pearson Educación.

Brun, X., Larraga, P., \& Moya, M. (2010). Cómo interpretar la información económica: Análisis de mercados financieros: coyuntura económica, sistema financiero, política monetar. Barcelona : Profit Editorial.

Carvalho, C. (2013). Teoría de la Decisión Tributaria. Perú: Saraiva - São Paulo.

Chirinos, C. (2009). Las campanas suenan, pero nadie las escucha: las exoneraciones y el IGV. Revista de Economía y Derecho, 6(24), 69-92.

Decreto Supremo N 055-99 - EF (2006). Texto Único Ordenado la ley del Impuesto General a las Ventas e Impuesto Selectivo al Consumo (15 de Abril de 1999). Diario Oficial El Peruano.

Flores, J. (2013). Flujo de Caja. Perú: Pacifico Editores.

Hax, A., \& Majluf, N. (2004). Estrategias para el liderazgo competitivo. Buenos Aires: Ediciones Granica.

Hernández , R., Fernández, C., \& Baptista, P. (2014). Metodología de la investigación (Sexta ed.). México: MgGRAW-HILL.

Ibáñez, J. (2015). Métodos, técnicas e instrumentos de la investigación criminológica. Madrid: Dikynson S.L.

Jaramillo, H., \& Tovar, J. (2010). Reflexiones sobre la teoría y práctica del IVA en Colombia. Revista de Economía del Rosario, 10(2), 171-188.

Mostacero, D. (2018). Informalidad en las MYPES y su Influencia en la recaudación del Impuesto General a las Ventas. QUIPUKAMAYOC, 26(50); 41-50. Obtenido de https://doi.org/10.15381/quipu.v26i50.14723

Palella, S., \& Martins, F. (2012). Metodología de la investigación cuantitativa (Tercera ed.). Venezuela: Fondo Editorial Universidad Pedagógica Experimental Libertador.

Peralta, E. (2016). Teoría General de los Sistemas aplicada a modelos de Gestión. Aglala, 7(1), 122-146. doi:10.22519/22157360.901 
Puente, A. (2006). Finanzas corporativas para el Perú. Lima: Pacífico Editores.

Rodríguez, A. (2014). Estrategias de planificación financiera aplicada. Santiago de Compostela: Andavira Editora.

Rojas , R. (2011). Guía para realizar investigaciones sociales. México: Plaza y Valdés.

SUNAT. (02 de Agosto de 2017). Emprender. Obtenido de http://emprender.sunat.gob.pe/igvjusto

SUNAT. (14 de Agosto de 2018). Emprende. Obtenido de http://emprender.sunat.gob.pe/pagode-mis-tributos

SUNAT. Orientacion SUNAT. Obtenido de http://orientacion.sunat.gob.pe/index.php/empresas-menu/impuesto-general-a-las-ventasy-selectivo-al-consumo/impuesto-general-a-las-ventas-igv-empresas/3109-05-calculodel-impuesto. 